\title{
Características clínicas y tomográficas asociadas al manejo quirúrgico en pacientes con obstrucción intestinal de origen adherencial
}

\author{
Clinical and tomographic features associated with surgical management in adhesive \\ small bowel obstruction patients
}

\author{
Julián G. Toto-Morales*, Ángel Martínez-Munive y Fernando Quijano-Orvañanos \\ Departamento de Cirugía General, Centro Médico ABC, Ciudad de México, México
}

\begin{abstract}
Resumen
Antecedentes: El retraso en el manejo quirúrgico de los pacientes con oclusión intestinal aumenta la morbimortalidad y los días de estancia intrahospitalaria. Objetivo: Describir las características clínicas y tomográficas de ingreso asociadas al manejo quirúrgico en pacientes con oclusión intestinal adherencial. Método: Revisamos los expedientes electrónicos de pacientes de los últimos 5 años con diagnóstico de oclusión intestinal adherencial y los dividimos según recibieran tratamiento conservador o tratamiento quirúrgico, y comparamos las variables. Resultados: Cumplieron los criterios de inclusión 162 pacientes, con una edad media de 61.5 años. El episodio capturado fue el primer episodio de oclusión intestinal en el $63 \%$ de los pacientes. Se realizó tratamiento quirúrgico al $65.4 \%$ de los pacientes $(n=106): 52.8 \%(n=56)$ cirugía abierta y $47.2 \%$ $(n=50)$ cirugía laparoscópica. El análisis multivariado mostró los siguientes factores predictores de tratamiento quirúrgico: presencia de rebote abdominal (odds ratio [OR]: 8.8; intervalo de confianza del 95\% [IC95\%]: 1.09-71.6), líquido libre (OR: 4.62; IC95\%: 1.50-14.20) y zona de transición por tomografía (OR: 5.4; IC95\%: 1.59-18.80). El antecedente de oclusiones previas fue un factor protector (OR: 0.33; IC95\%: 0.17-0.67). Conclusiones: El rebote abdominal, el líquido libre y la presencia de zona de transición por tomografía están asociados al manejo quirúrgico en los pacientes con oclusión intestinal adherencial.
\end{abstract}

Palabras clave: Obstrucción de intestino delgado. Cirugía. Adherencias.

\begin{abstract}
Background: The delay in surgical management of intestinal obstruction patients who did not respond to conservative management increases morbidity, mortality and days of hospital stay. Objective: This study aimed to describe the clinical and tomographic features associated with surgical management in adhesive small bowel obstruction patients. Method: We conducted a retrospective review of the electronic medical records during a 5-year period with the diagnosis of adhesive small bowel obstruction. We divided patients in two, those who responded to medical management and those who required surgery. Results: A total of 162 patients were included, with a mean age of 61.5 years. It was the first case of intestinal obstruction in $63 \%$ of the patients and $65.4 \%$ underwent surgery: $52.8 \%(n=56)$ open surgery and $47.2 \%(n=50)$ laparoscopic surgery.
\end{abstract}

\footnotetext{
Correspondencia:

*Julián G. Toto-Morales

Sur 136, 116

Las Américas, Del. Álvaro Obregón

Fecha de recepción: 01-07-2020

Cir Cir. 2021;89(5):588-594

C.P. 01120 , Ciudad de México, México

Fecha de aceptación: 09-12-2020

Contents available at PubMed

E-mail: drjuliantoto@gmail.com

DOI: 10.24875/CIRU.20000716

www.cirugiaycirujanos.com

0009-7411/@ 2020 Academia Mexicana de Cirugía. Publicado por Permanyer. Este es un artículo open access bajo la licencia CC BY-NC-ND (http://creativecommons.org/licenses/by-nc-nd/4.0/).
} 
Multivariate analysis showed the following predictors of surgical treatment: abdominal rebound (odds ratio [OR]: 8.8; 95\% confidence interval [95\% Cl]: 1.09-71.6), tomographic free fluid (OR: 4.62; $95 \% \mathrm{Cl}: 1.50-14.20$ ) and transition zone (OR: 5.4; 95\% Cl: 1.59-18.80). The history of previous obstruction was a protective factor (OR: $0.33 ; 95 \% \mathrm{Cl}: 0.17-0.67$ ). Conclusions: Abdominal rebound, free intrabdominal fluid and transition zone are related with the surgical management of adhesive small bowel obstruction.

Key words: Small bowel obstruction. Surgery. Adhesions.

\section{Introducción}

La atención de pacientes con oclusión intestinal es una tarea muy frecuente en los servicios quirúrgicos, como una de las primeras causas de dolor abdominal agudo ${ }^{1}$. Alrededor del $7 \%$ de los pacientes presentarán un cuadro de oclusión intestinal tras su primera cirugía abdominal y se estima que al menos el $25 \%$ lo harán en el primer año². Aunque la mayoría responderán al manejo conservador, el retraso en la cirugía de aquellos que finalmente no respondan aumenta la morbimortalidad y los días de estancia hospitalaria ${ }^{3,4}$. Se ha reportado una menor mortalidad, así como una menor tasa de recurrencia de cuadros oclusivos, en los pacientes tratados quirúrgicamente en comparación con los manejados con tratamiento conservador ${ }^{5.7}$, por lo que nos dimos a la tarea de investigar aquellas características clínicas y tomográficas asociadas al tratamiento quirúrgico en los pacientes con oclusión intestinal al momento de su ingreso, en un intento por caracterizar a este subgrupo de pacientes.

\section{Método}

Se trata de un estudio de cohorte retrospectivo de 5 años en el que se incluyeron todos los pacientes mayores de 18 años con diagnóstico de oclusión intestinal adherencial (CIE-9 54.5, 560.81, 560.9; CIE10 56.5) y expediente electrónico completo, que fueron divididos en dos grupos según hubieran recibido tratamiento médico conservador o tratamiento quirúrgico. Se excluyeron aquellos pacientes que habían sido operados en las 6 semanas previas a su ingreso, con cuadros oclusivos de etiología neoplásica o herniaria, por enfermedad inflamatoria intestinal o vólvulos, así como los pacientes con expedientes incompletos o que hubieran sido trasladados a otros centros hospitalarios antes de conocer el desenlace del manejo.

El objetivo primario fue el desenlace quirúrgico, definido como aquellos pacientes que se sometieron a cirugía a su ingreso o tras un periodo de manejo médico conservador. Las variables clínicas recolectadas fueron la edad, el sexo, la fecha de ingreso, la fecha de la cirugía, el número de cirugías abdominales previas al episodio de oclusión intestinal en cuestión, el tipo de cirugías abdominales y el número de episodios previos de oclusión intestinal referidos en la historia clínica. Se decidió incluir a todo paciente con diagnóstico de oclusión intestinal sin antecedente de cirugía abdominal previa. Se documentó la presencia de comorbilidad (diabetes mellitus, hipertensión arterial, cardiopatía y hepatopatía). Los hallazgos de la exploración física registrados al momento del ingreso fueron la presencia de signo de rebote, fiebre $\left(>38^{\circ} \mathrm{C}\right)$, frecuencia cardiaca y presencia de síndrome de respuesta inflamatoria sistémica, definido por el consenso del American College of Chest Physicians y la Society of Critical Care Medicine ${ }^{8}$ como la presencia de dos o más de los siguientes parámetros: temperatura $>38{ }^{\circ} \mathrm{C} 0<36{ }^{\circ} \mathrm{C}$, frecuencia cardiaca $>90$ latidos por minuto, taquipnea $>20$ y leucocitos $>12.0000<4.000$. El gasto de apertura de la sonda se definió como el gasto obtenido inmediatamente después de su colocación en el servicio de urgencias o en hospitalización. Las variables de laboratorio incluidas para el análisis fueron los leucocitos, el hematocrito, la albúmina, el lactato y la proteína $C$ reactiva ultrasensible.

Se revisaron las tomografías de ingreso y se analizaron la presencia de zona de transición, el diámetro máximo del lumen intestinal previo a la zona de transición, la presencia de líquido libre en la cavidad peritoneal y el signo de las heces o fecalización del intestino delgado. Las imágenes fueron revisadas por un médico radiólogo.

Realizamos estadística descriptiva con medidas de tendencia central y de dispersión. Las variables categóricas se expresan como frecuencias absolutas y relativas, y las variables lineales como media y desviación estándar o mediana y rangos intercuartilares según corresponda a la distribución de frecuencias. La estadística inferencial incluyó el uso de pruebas 
de hipótesis para evaluar variables lineales con la prueba t de Student para muestras relacionadas. Las variables categóricas se analizaron con la prueba de ji al cuadrado. Se realizó un análisis de sensibilidad y especificidad para buscar el punto de corte del gasto de apertura de la sonda nasogástrica que predijera el desenlace quirúrgico. La medida de la fuerza de la asociación utilizada fue la razón de riesgo con un intervalo de confianza del 95\% (IC95\%) utilizando como variable de desenlace el requerir abordaje quirúrgico, y se calculó mediante estratificación de edad y sexo con el método Cochran-Mantel-Haenszel; además, se construyeron modelos de regresión logística binaria tomando en cuenta el mismo desenlace utilizando como variables independientes criterios clínicos de ingreso. Se consideró una $p>0.05$ como significativa a dos colas para todas las pruebas de hipótesis. La paquetería estadística fue STATA SE 13.0

\section{Aspectos éticos}

El proyecto cumple la normativa internacional y local de estudios de investigación en humanos, acorde con la Ley General de Salud y la Declaración de HeIsinki. Se le considera como investigación sin riesgo por obtención de datos a través de la revisión de expedientes clínicos.

\section{Resultados}

Se revisaron los expedientes de 263 pacientes, de los cuales 162 cumplieron los criterios de inclusión (Fig. 1), con una media de edad de $61.5( \pm 15)$ años y un $52 \%$ de pacientes del sexo femenino $(n=84)$. $\mathrm{Se}$ registraron las siguientes enfermedades como comorbilidad: hipertensión arterial sistémica en el $36 \%(n=59)$, cardiopatía en el $17 \%(n=28)$ y diabetes mellitus en el $6.8 \%(n=11)$. El $7.4 \%$ de los pacientes no tenían antecedentes quirúrgicos abdominales en el primer episodio de oclusión $(n=12)$, el $49.4 \%(n=80)$ tenían antecedente de solo una cirugía abdominal previa y el $43.25 \%(n=70)$ tenían antecedente de dos o más intervenciones quirúrgicas abdominales (Fig. 2). El episodio capturado fue el primer episodio de oclusión intestinal en el $63 \%$ de los pacientes $(n=102)$. El $37 \%$ de los pacientes $(n=60)$ informaron haber presentado al menos un episodio previo, y de estos, en el $43 \%$ ( $n=26 / 60)$ fue su tercer episodio o más (Fig. 3). Entre los sujetos con antecedentes quirúrgicos abdominales

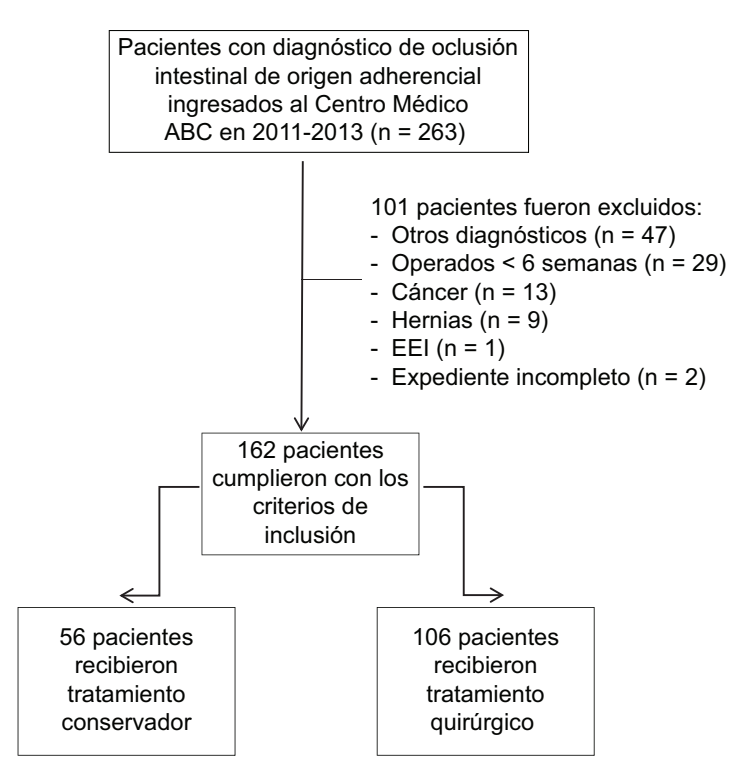

Figura 1. Diagrama de flujo de la inclusión de pacientes. EEl: Enfermedad Inflamatoria Intestinal.

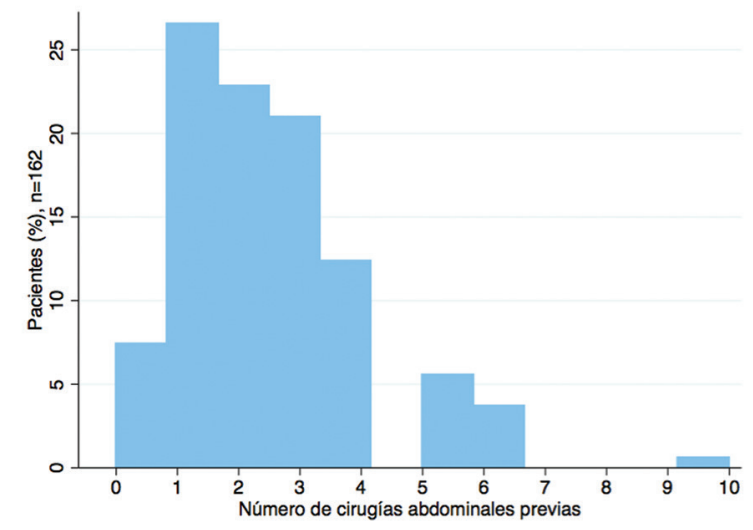

Figura 2. Histograma con el porcentaje de pacientes según el número de cirugías abdominales previas en sujetos ingresados por oclusión intestinal adherencial. La mayoría de los pacientes tenían antecedentes quirúrgicos abdominales; la moda fue de 1 episodio.

( $n=150)$ se compararon aquellos con dos o más cirugías abdominales frente a los que solo tuvieron una cirugía previa, sin observar diferencia en la proporción de pacientes que se sometieron a cirugía y aquellos que recibieron manejo conservador ( $67.5 \mathrm{vs.}$ $60 \% ; p=0.340$ ). Las frecuencias de los principales tipos de cirugías abdominales previas al cuadro oclusivo se muestran en la tabla 1. La cirugía ginecológica fue la más frecuente en términos generales, seguida de la apendicectomía, la colecistectomía y la cirugía de colon. En el grupo de tratamiento conservador se observó una mayor proporción de 


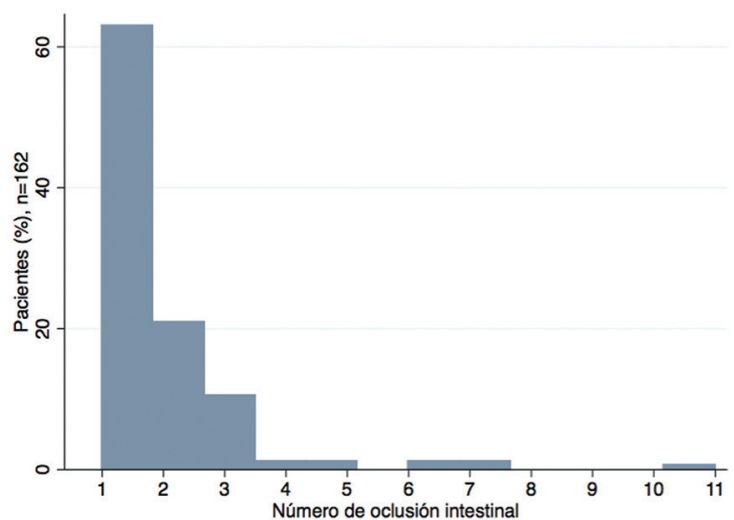

Figura 3. Histograma con el porcentaje de pacientes según el número de episodios de oclusión abdominal adherencial al ingreso. La mayor proporción de pacientes se encontraban cursando su primer episodio de oclusión (63\%).

Tabla 1. Principales tipos de cirugías en pacientes con oclusión intestinal $(n=150)$

\begin{tabular}{lcc}
\hline Cirugía & Frecuencia & Porcentaje* $^{*}$ \\
\hline Colecistectomía & 49 & 32.7 \\
Cirugía de colon & 43 & 28.7 \\
Apendicectomía & 52 & 34.7 \\
Ginecológica (solo mujeres, $\mathrm{n}=80)$ & 80 & 79.3 \\
Otras & 65 & 43.3 \\
\hline
\end{tabular}

*Los pacientes pudieron haber tenido más de una cirugía previa, por lo que el porcentaje total no suma el $100 \%$.

antecedentes de oclusión intestinal. El grupo quirúrgico presentó una mayor frecuencia de signo de rebote, más días de estancia hospitalaria y una mayor tasa de líquido libre y de zona de transición en la tomografía (Tablas 2 y 3). Se realizó tratamiento quirúrgico al $65.4 \%$ de los pacientes $(n=106)$, con el siguiente abordaje: en el $52.8 \%(n=56)$ cirugía abierta y en el $47.2 \%(n=50)$ cirugía laparoscópica. De estos últimos, el $38 \%(n=19)$ se convirtieron a cirugía abierta. Al realizar el cálculo del riesgo relativo utilizando como variable dependiente la realización de procedimiento quirúrgico y estratificar a los pacientes por edad mayor o menor de 65 años, observamos que haber tenido antecedentes de oclusión intestinal fue un factor protector de recibir tratamiento quirúrgico durante la hospitalización, protección que se reprodujo solo en los mayores de 65 años. Las variables tomográficas de riesgo fueron la zona de transición y el líquido libre (Tabla 4).
Tabla 2. Comparación de las características generales de los pacientes con oclusión intestinal adherencial en el grupo con tratamiento conservador y en el grupo con tratamiento quirúrgico

\begin{tabular}{|c|c|c|c|c|}
\hline & $\begin{array}{c}\text { Total } \\
(n=162)\end{array}$ & $\begin{array}{c}\text { Tratamiento } \\
\text { conservador } \\
(n=56)\end{array}$ & $\begin{array}{l}\text { Tratamiento } \\
\text { quirúrgico } \\
(n=106)\end{array}$ & 0 \\
\hline \multicolumn{5}{|l|}{ Antecedentes médicos } \\
\hline Sexo femenino, n (\%) & $84(51.9)$ & $31(55.4)$ & $47(44.3)$ & 0.182 \\
\hline $\begin{array}{l}\text { Edad en años, media } \\
\text { (DE) }\end{array}$ & 61.5 & $63.3(14.1)$ & $60.6(16.2)$ & 0.286 \\
\hline $\begin{array}{l}\text { Diabetes mellitus, } \\
\mathrm{n}(\%)\end{array}$ & $11(6.8)$ & $5(8.3)$ & $6(5.6)$ & 0.432 \\
\hline $\begin{array}{l}\text { Hipertensión arterial } \\
\text { sistémica, } \mathrm{n}(\%)\end{array}$ & $59(36.4)$ & $23(41.1)$ & $36(33.9)$ & 0.371 \\
\hline Cardiopatía, n (\%) & 28 (17.3) & $13(23.2)$ & $15(14.2)$ & 0.147 \\
\hline $\begin{array}{l}\text { Cirugía abdominal } \\
\text { previa al primer } \\
\text { episodio de oclusión, } \\
\text { n (\%) }\end{array}$ & $150(92.6)$ & $54(96.4)$ & $96(90.6)$ & 0.175 \\
\hline $\begin{array}{l}\text { Dos o más cirugías } \\
\text { abdominales previas } \\
\text { al primer episodio de } \\
\text { oclusión, } n(\%)\end{array}$ & $70(43.2)$ & $28(50)$ & $42(39.6)$ & 0.205 \\
\hline $\begin{array}{l}\text { Episodios previos de } \\
\text { oclusión, } n(\%)\end{array}$ & $60(37)$ & $30(53.6)$ & $30(28.3)$ & 0.002 \\
\hline $\begin{array}{l}\text { Dos o más episodios } \\
\text { previos de oclusión, } \\
\text { n (\%) }\end{array}$ & $26(16.1)$ & $19(33.4)$ & $7(6.6)$ & $<0.001$ \\
\hline \multicolumn{5}{|l|}{$\begin{array}{l}\text { Cuadro clínico al } \\
\text { ingreso }\end{array}$} \\
\hline $\begin{array}{l}\text { Síndrome de } \\
\text { respuesta inflamatoria } \\
\text { sistémica, } \mathrm{n}(\%)\end{array}$ & $a^{14(8.8)}$ & $2(3.6)$ & $12(11.5)$ & 0.089 \\
\hline Fiebre, n (\%) & $2(1.2)$ & $1(1.8)$ & $1(1.8)$ & 0.644 \\
\hline Taquicardia, n (\%) & $12(7.4)$ & $3(5.4)$ & $9(8.5)$ & 0.469 \\
\hline Rebote, $\mathrm{n}(\%)$ & $16(9.9)$ & $1(1.8)$ & $15(14.2)$ & 0.012 \\
\hline Gasto de apertura de & & & & \\
\hline $\begin{array}{l}\text { la sonda nasogástrica } \\
(\mathrm{ml}), \text { media }(\mathrm{DE}) \\
(\mathrm{n}=129)\end{array}$ & a $193(340)$ & $128(35.8)$ & $222(39.8)$ & 0.153 \\
\hline $\begin{array}{l}\text { Días de estancia } \\
\text { hospitalaria, media } \\
\text { (DE) }\end{array}$ & $7.2(6.3)$ & $4.2(3.2)$ & $8.9(6.1)$ & $<0.001$ \\
\hline
\end{tabular}

DE: desviación estándar.

El análisis multivariado mostró que el factor clínico de ingreso que predijo que el paciente recibiera tratamiento quirúrgico fue la presencia de rebote abdominal (odds ratio [OR]: 8.8; IC95\%: 1.09-71.6), independientemente de la presencia de síndrome de respuesta inflamatoria sistémica y del gasto de apertura de la sonda nasogástrica al ingreso. El antecedente de oclusiones previas fue un factor protector (OR: 0.33; IC95\%: 0.17-0.67). El análisis multivariado mostró que los hallazgos tomográficos predictores de recibir tratamiento quirúrgico fueron el líquido libre (OR: 4.62; IC95\%: 1.50-14.20) y la zona de transición (OR: 5.4; IC95\%: 1.59-18.80). 
Tabla 3. Estudios de laboratorio y de gabinete en pacientes con oclusión intestinal adherencial en el grupo de tratamiento conservador y en el grupo de tratamiento quirúrgico

\begin{tabular}{|c|c|c|c|c|}
\hline & Total & $\begin{array}{l}\text { Tratamiento conservador } \\
\qquad(\mathrm{n}=56)\end{array}$ & $\begin{array}{l}\text { Tratamiento } \\
\text { quirúrgico }(n=106)\end{array}$ & $\mathbf{p}$ \\
\hline \multicolumn{5}{|l|}{ Estudios de laboratorio, media (DE) } \\
\hline Leucocitos $\left(10^{3} / \mu \mathrm{l}\right)$ & $10.471(3814)(n=162)$ & $9894(3451)(n=56)$ & $10,776(3974)(n=56)$ & 0.162 \\
\hline Hematocrito (\%) & $45.8(6)(n=162)$ & $45(6.1)(n=162)$ & $46(5.9)(n=162)$ & 0.230 \\
\hline Albúmina (mg/dl) & $4.2(0.57)(n=124)$ & $4.1(0.53)(n=44)$ & $4.3(0.57)(n=88)$ & 0.058 \\
\hline Deshidrogenasa láctica (mg/dl) & $185(57)(n=15)$ & $172(73)(n=4)$ & $190(65)(n=11)$ & 0.603 \\
\hline Lactato $(\mathrm{mmol} / \mathrm{l})$ & $1.96(1.1)(n=61)$ & $1.71(0.78)(n=9)$ & $2.0(1.13)(n=52)$ & 0.463 \\
\hline Proteína $\mathrm{C}$ reactiva ultrasensible $(\mathrm{mg} / \mathrm{dl})$ & $2.8(5)(n=82)$ & $2.3(2.9)(n=25)$ & $3.1(5.6)(n=57)$ & 0.525 \\
\hline \multicolumn{5}{|l|}{ Hallazgos tomográficos ( $n=96)$} \\
\hline Líquido libre & $63.4(61)$ & $12(42.3)$ & $61(63.5)$ & 0.007 \\
\hline Zona de transición & $97(80.8)$ & $23(60.5)$ & $70(90.2)$ & $<0.001$ \\
\hline Fecalización & $43(53.7)$ & $15(62.5)$ & $28(50)$ & 0.304 \\
\hline
\end{tabular}

Tabla 4. Riesgo relativo de requerir cirugía durante la hospitalización en pacientes con oclusión intestinal adherencial estratificado por edad

\begin{tabular}{|c|c|c|c|c|}
\hline & RR total (IC95\%) & RR < 65 años (IC 95\%) & $R R \geq 65$ años (IC95\%) & $p^{*}$ \\
\hline \multicolumn{5}{|l|}{ Variables clínicas } \\
\hline Diabetes mellitus tipo 2 & $0.82(0.47-1.43)$ & - & $0.99(0.57-1.71)$ & 0.650 \\
\hline Hipertensión arterial sistémica & $0.90(0.70-1.45)$ & $0.87(0.58-1.31)$ & $1.00(0.68-1.47)$ & 0.620 \\
\hline Cardiopatía & $0.79(0.55-1.13)$ & $0.70(0.31-1.58)$ & $0.86(0.56-1.34)$ & 0.650 \\
\hline Cirugía abdominal previa & $0.77(0.58-1.01)$ & $0.67(0.58-0.78)$ & $(0.82-0.49-1.37)$ & 0.159 \\
\hline Oclusión intestinal previa & $0.67(0.51-0.89)$ & $0.91(0.67-1.24)$ & $0.45(0.27-0.74)$ & 0.013 \\
\hline Rebote abdominal & $1.5(1.26-1.80)$ & $1.5(1.3-1.80)$ & $1.36(0.84-2.2)$ & 0.592 \\
\hline Gasto de la sonda $\geq 250 \mathrm{ml}(\mathrm{n}=129)$ & $1.02(0.81-1.27)$ & $0.84(0.61-1.16)$ & $1.38(0.93-2.05)$ & 0.057 \\
\hline Síndrome de respuesta inflamatoria sistémica & $1.36(1.06-1.74)$ & $1.26(0.90-1.78)$ & $1.48(1.03-2.14)$ & 0.532 \\
\hline Leucocitosis o leucocitopenia & $1.01(0.80-1.29)$ & $0.89(0.64-1.22)$ & $1.19(0.82-11.73)$ & 0.234 \\
\hline Lactato elevado $(n=61)$ & $0.98(0.79-1.21)$ & $1.14(0.88-1.48)$ & $0.80(0.56-1.14)$ & 0.111 \\
\hline \multicolumn{5}{|l|}{ Variables tomográficas $(n=96)$} \\
\hline Zona de transición & $2.19(1.24-3.89)$ & $3.45(1.26-9.37)$ & $1.44(0.75-2.75)$ & 0.121 \\
\hline Líquido libre & $1.48(1.07-2.05)$ & $1.56(0.89-2.27)$ & $1.56(0.99-2.47)$ & 0.778 \\
\hline Fecalización & $0.86(0.65-1.15)$ & $0.75(0.51-1.12)$ & $1.03(0.69-1.55)$ & 0.255 \\
\hline
\end{tabular}

*Obtenido de la diferencia de proporciones entre ambas columnas.

IC95\%: intervalo de confianza del 95\%; RR: razón de riesgo.

\section{Discusión}

Los resultados demográficos de nuestro estudio están a la par con los de otras series, en las que el promedio de edad ronda los 60 años y con un claro predominio del sexo femenino $0^{5,9,10}$, sin que hasta el momento haya evidencia de que ser mujer sea un factor de riesgo independiente de oclusión intestinal ${ }^{11}$. Lo anterior podría explicarse por datos demográficos: en nuestro país, las mujeres predominan en número, y según cifras del Instituto Mexicano del Seguro Social, las mujeres acaparan hasta el $70 \%$ del total de las cirugías, sobre todo en edades medias ${ }^{12}$. Dos tercios de los pacientes nunca habían presentado cuadros oclusivos previos y la gran mayoría tenían antecedente de al menos una cirugía abdominal, lo que concuerda con lo reportado por O'Leary, et al. ${ }^{13}$ y es congruente con el conocimiento de que las adherencias (inevitablemente formadas tras una cirugía) son la causa directa de este padecimiento ${ }^{14}$. En nuestra serie, solo el $7.4 \%$ de los pacientes no tenían antecedentes de cirugías abdominales, mientras que la mayoría (49\%) solo tenían una laparotomía previa, de modo similar a lo reportado en algunos estudio ${ }^{15,16} \mathrm{y}$ que señala que la probabilidad de cuadros oclusivos no depende del número de laparotomías previas. Entre el $30 \%$ y el $40 \%$ de los pacientes con oclusión intestinal han presentado cuadros oclusivos previos $^{15-17}$, cifra que concuerda con el $37 \%$ de nuestra serie. En este caso, el número de episodios previos 
de oclusión (a diferencia del número de laparotomías) sí parece influir en el riesgo de recurrencia, siendo mayor el riesgo cuanto mayor es el número de cuadros previos $6,15,18$.

En la serie de Komatsu, et al. ${ }^{19}$, el grupo quirúrgico presentó un mayor promedio de laparotomías previas en el análisis univariado, resultado que no fue significativo en el análisis multivariado. En nuestra serie no hubo diferencia en la frecuencia de cirugías previas en ambos grupos, situación que debilita la idea de que, a mayor número de cirugías previas, menor respuesta al manejo conservador. En relación con el tipo de cirugía, nuestro hallazgo de la cirugía ginecológica como el tipo más frecuente concuerda con lo reportado por Barmparas, et al. ${ }^{11}$, quienes en una revisión de casi 450,000 cirugías abdominales reportaron que la incidencia más alta de cuadros oclusivos adherenciales la encontraron en el grupo de pacientes con cirugía abierta de anexos (23\%), seguida de las anastomosis ileorrectales con reservorio (19\%) y las histerectomías abdominales (15\%). Sin embargo, para Williams, et al. ${ }^{7}$ los procedimientos colorrectales son los más frecuentes, aunque hay que señalar que su serie de 321 pacientes corresponde a un departamento de cirugía colorrectal. Por otro lado, no encontramos diferencias entre los grupos respecto a los tipos de cirugías previas, tal como reportan Meier, et al. ${ }^{6}$ en un análisis retrospectivo de 221 pacientes con oclusión. Dos tercios de los pacientes requirieron tratamiento quirúrgico, prácticamente invirtiéndose los papeles de aquellas series en que la resolución espontánea alcanza un $70-80 \% 5,20,21$, aunque en la literatura pueden observarse series con tasas de manejo quirúrgico similares ${ }^{6,7}$.

El único dato clínico al ingreso asociado al manejo quirúrgico en nuestra serie fue la presencia del signo de rebote; sin embargo, cuando se excluyen los pacientes con datos de estrangulación al ingreso, algunos estudios no reportan datos clínicos o de laboratorio asociados al manejo quirúrgico ${ }^{19-22}$, mientras que otros indican la ausencia de canalización de gases ${ }^{17}$ o una elevada concentración de creatina fosfocinasa en sangre ${ }^{16}$ como posibles indicadores de cirugía. Zielinski, et al. ${ }^{23}$ señalan que el vómito es un predictor de tratamiento quirúrgico, pero se trata de una serie no exclusiva de pacientes con oclusión adherencial. Mientras que no hubo diferencias en nuestra serie en cuanto al gasto de apertura de la sonda nasogástrica, Komatsu, et al. ${ }^{19}$ reportan que un gasto $>500 \mathrm{ml}$ al tercer día es un factor crítico que predice el manejo quirúrgico.
En relación con los hallazgos tomográficos, existen conclusiones variadas. Al igual que en nuestro estudio, la presencia de líquido libre en otras series ${ }^{17,19,24}$ se ha asociado con la necesidad de manejo quirúrgico, sobre todo en pacientes que presentan líquido libre de alta densidad ${ }^{25}$. Hwang, et al. ${ }^{26}$, al igual que nosotros, reportan la zona de transición como un predictor de tratamiento quirúrgico. Aunque no fue evaluado en nuestra serie, vale la pena mencionar que muchos autores ${ }^{16,17,24,26}$ concuerdan en que la presencia de una obstrucción de alto grado (ausencia de gas o líquido en el colon) también es un indicador de tratamiento quirúrgico. Finalmente, mientras que Zielinski, et al. ${ }^{23}$ reportaron en su análisis univariado el antecedente de cuadros oclusivos como un factor asociado al manejo conservador exitoso, nuestro análisis multivariado lo señala como un factor protector a pesar de que la prevalencia de cuadros oclusivos previos es similar a la reportado en la literatura.

Las limitantes de nuestro estudio inician por su naturaleza retrospectiva y por ser unicéntrico, lo que genera un potencial sesgo de selección, y a lo anterior hay que agregar el hecho de que los casos son manejados por distintos cirujanos y médicos internistas, lo que impacta en el abordaje y el manejo de cada paciente a pesar de las recomendaciones internacionales existentes. Sin embargo, creemos que los resultados pueden contribuir a concientizar sobre la necesidad de realizar un abordaje sistematizado apegado a las guías internacionales, y así mismo colaborar en el proceso de toma de decisiones ante un paciente con diagnóstico de oclusión intestinal.

\section{Conclusiones}

El signo del rebote y la presencia de zona de transición y líquido libre en la tomografía de ingreso de los pacientes con oclusión intestinal adherencial estuvieron asociados al manejo quirúrgico. Las pruebas de laboratorios de ingreso y el gasto de apertura de la sonda nasogástrica no estuvieron asociados al manejo quirúrgico. El antecedente de oclusiones intestinales previas sí está asociado a una menor necesidad de tratamiento quirúrgico.

\section{Agradecimientos}

Los autores agradecen al Dr. Fernando Quijano Orvañanos su valiosa cooperación en la planeación y la elaboración de este trabajo. 


\section{Conflicto de intereses}

Los autores declaran no tener conflictos de intereses.

\section{Responsabilidades éticas}

Protección de personas y animales. Los autores declaran que para esta investigación no se han realizado experimentos en seres humanos ni en animales.

Confidencialidad de los datos. Los autores declaran que han seguido los protocolos de su centro de trabajo sobre la publicación de datos de pacientes.

Derecho a la privacidad y consentimiento informado. Los autores declaran que en este artículo no aparecen datos de pacientes.

\section{Bibliografía}

1. Eskelinen M, Ikonen J, Lipponen P, Central S. Contributions of history-taking, physical examination, and computer assistance to diagnosis of acute small-bowel obstruction: a prospective study of 1333 patients with acute abdominal pain. Scand J Gastroenterol. 1994;29:715-21.

2. Parker MC, Ellis H, Moran BJ, Thompson JN, Wilson MS, Menzies D, et al. Postoperative adhesions: ten-year follow-up of 12,584 patients undergoing lower abdominal surgery. Dis Colon Rectum. 2001;44:822-9.

3. Fevang BT, Fevang J, Stangeland L, Søreide O. Complications and death after surgical treatment of small bowel obstruction. Ann Surg. 2000;231:529-37.

4. Keenan JE, Turley RS, McCoy CC, Migaly J, Shapiro ML, Scarborough JE, et al. Trials of nonoperative management exceeding 3 days are associated with increased morbidity in patients undergoing surgery for uncomplicated adhesive small bowel obstruction. J Trauma Acute Care Surg. 2014;76:1367-72.

5. Foster NM, Mcgory ML, Zingmond DS, Ko CY. Small bowel obstruction: a population-based appraisal. J Am Coll Surg. 2006:203:170-6.

6. Meier RPH, Oulhaci W, Orci LA, Gutzwiller EM, Fre PM. Clinical outcome in acute small bowel obstruction after surgical or conservative management. World J Surg. 2014;38:3082-8.

7. Williams SB, Greenspon J, Young HA, Orkin BA. Small bowel obstruction: conservative vs. surgical management. Dis Colon Rectum. 2005;48:1140-6.

8. Bone RC, Balk RA, Cerra FB, Dellinger RP, Fein AM, Knaus WA, et al. American College of Chest Physicians/Society of Critical Care Medicine Consensus Conference: definitions for sepsis and organ failure and guidelines for the use of innovative therapies in sepsis. Chest. 1992;101:1644-55.
9. De la Garza-Villaseñor L. Etiología de la oclusión intestinal. Rev Gastroenterol Mex. 2001;66:193-6.

10. Markogiannakis H, Messaris E, Dardamanis D, Pararas N, Tzertzemelis D, Giannopoulos P, et al. Acute mechanical bowel obstruction: clinical presentation, etiology, management and outcome. World J Gastroenterol. 2007;13:432-7.

11. Barmparas G, Branco BC, Schnüriger B, Lam L, Inaba K, Demetriades D. The incidence and risk factors of post-laparotomy adhesive small bowel obstruction. J Gastrointest Surg. 2010;14:1619-28.

12. Fernández-Cantón S. El IMSS en cifras. Las intervenciones quirúrgicas. Rev Med Inst Mex Seguro Soc. 2005;43:511-20.

13. O'Leary EA, Desale SY, Yi WS, Fujita KA, Hynes CF, Chandra SK, et al. Letting the sun set on small bowel obstruction: can a simple risk score tell us when nonoperative care is inappropriate ? Am Surg. 2014;80:572-9.

14. Menzies D, Ellis $H$. Intestinal obstruction from adhesions - how big is the problem? Ann R Coll Surg Engl. 1990;72:60-3.

15. Miller G, Boman J, Shrier I, Gordon PH. Natural history of patients with adhesive small bowel obstruction. Br J Surg. 2000;87:1240-7.

16. Tanaka S, Yamamoto T, Kubota D, Matsuyama M, Uenishi T, Kubo S, et al. Predictive factors for surgical indication in adhesive small bowel obstruction. Am J Surg. 2008;196:23-7.

17. Kulvatunyou N, Pandit V, Moutamn S, Inaba K, Chouliaras K, Demoya M, et al. A multi-institution prospective observational study of small bowel obstruction: clinical and computerized tomography predictors of which patients may require early surgery. J Trauma Acute Care Surg. 2015;79:393-8.

18. Fevang J, Lie SA, Svanes K, Viste A. Long-term prognosis after operation for adhesive small bowel obstruction. Ann Surg. 2004;240:193-201.

19. Komatsu I, Tokuda Y, Shimada G, Jacobs JL, Onodera H. Development of a simple model for predicting need for surgery in patients who initially undergo conservative management for adhesive small bowel obstruction. Am J Surg. 2010;200:215-23.

20. Menzies D, Parker M, Hoare R, Knight A. Small bowel obstruction due to postoperative adhesions: treatment patterns and associated costs in 110 hospital admissions. Ann R Coll Surg Engl. 2001;83:40.

21. Schraufnagel D, Rajaee S, Millham FH. How many sunsets? Timing of surgery in adhesive small bowel obstruction: a study of the nationwide inpatient sample. J Trauma Acute Care Surg. 2012;74:181-9.

22. Kubasiak J, Blears E, Veenstra B, Francescatti A, Myers J, Millikan KW, et al. Factors that predict failure of non-operative management of small bowel obstructions. Gastroenterology. 2014;146:S-1094.

23. Zielinski MD, Eiken PW, Bannon MP, Heller SF, Lohse CM, Huebner M, et al. Small bowel obstruction - who needs an operation ? A multivariate prediction model. World J Surg. 2010;34:910-9.

24. Chang W, Ko K, Lin C, Hsu H, Tsai S, Fan H, et al. Features on MDCT that predict surgery in patients with adhesive-related small bowel obstruction. PLoS One. 2014;9:1-8.

25. Matsushima K, Inaba K, Dollbaum R, Cheng V, Khan M, Herr K, et al. High-density free fluid on computed tomography: a predictor of surgical intervention in patients with adhesive small bowel obstruction. J Gastrointest Surg. 2016;20:1861-6.

26. Hwang J-Y, Lee JK, Lee JE, Baek SY. Value of multidetector CT in decision making regarding surgery in patients with small-bowel obstruction due to adhesion. Eur Radiol. 2009;19:2425-31. 